\title{
IMPLEMENTATION OF P-TYPE BLACK SILICON WITH HIGH ASPECT RATIO FOR OPTOELECTRONICS APPLICATIONS
}

\author{
Hussam Muhsin Hwail $\bowtie$ \\ Department of Physics \\ University of Kufa \\ P.O. Box 21, Kufa, Al-Najaf, Iraq, 54007 \\ hussamm.daham@uokufa.edu.iq \\ Manal Midhat Abdullah \\ Department of Physics \\ University of Baghdad \\ Karrada, Al-Jadria, Baghdad, Iraq, 10071
}

$\triangle$ Corresponding author

\begin{abstract}
Black Silicon (BSi) is a semiconductor with a surface modified to get a very low reflectivity and correspondingly high absorption of visible light. P-type $<100>$ silicon wafers were used to prepare very low reflecting samples. These samples may use as substrates in optoelectronic applications. Electrochemical etching at different etching current, and time were applied. B-silicon is chemically equal to normal silicon, differs in surface treatment that changes a morphology. This special morphology is demanded for absorption enhancement which involves maximum light absorption. Surface roughness is done by texturing or trenching a silicon wafer surface. As these structures are in place, and as they are small enough, incident light will be more absorbed and less reflected, this is what gives black silicon its name. BSi was characterized by (FESEM), structural analysis indicates that BS layers were formed. The porosity was in the range of (10-40) \% and it is dependent on etching time and etching current value. Etching density is $0.1073 / \mathrm{mm}^{2}$. Reflection analysis shows satisfying minimum reflectivity spectrum with the intensity of peaks decrease and red shifted with increasing the etching current. A peak of the Photoluminescence ranges about $360 \mathrm{~nm}$, PL peaks are blue shifted and proportional to etching current. The band gap energy ranged from 2 to $3.4 \mathrm{eV}$, it is affected with porous morphology and directly proportional to etching current values.

Keywords: porous silicon, etching, energy gap, reflectivity, porosity, current, photoluminescence, black silicon, fabrication, morphology.
\end{abstract}

DOI: $10.21303 / 2461-4262.2021 .001902$

\section{Introduction}

Black Silicon (BSi) is a term given to low-reflectivity silicon wafer surface. This designation is based on the fact that the models have surface structures that allow high absorption and low reflectivity.

B-silicon is chemically equal to normal silicon, differs in the surface treatment that changes the morphology. This special morphology is demanded for absorption enhancement which involves maximum light absorption. Surface roughness is done by texturing or trenching the silicon wafer surface. When these structures are in place, and when they are small enough, incident light will be more absorbed and less reflected, this is what gives black silicon its name.

BSi has been applied to a wide range of applications such as micro-electro-mechanical systems (MEMS) chemical- and bio-sensors, optoelectronic and photonic device, bactericidal media, drug delivery, lithium-ion batteries. The nonporous layer has attracted considerable attention due to its visible light emissions and the large specific surface area of the material $[1,2]$.

The researchers recently studied porous Silicon (PS), which could be used in opto-electronic devices and sensors, intensively due to its photoluminescence (PL). The chemical agent of the silicon wafer was produced for this work, where the etchant comprises hydrofluoric acid (HF) and nitric acid. The etchant consists of $\left(\mathrm{HNO}_{3}\right)$. Scanning Electron Microscope (SEM) and Photoluminescence Specification Spectrometer characterized the PS [3, 4].

In the manufacturing of mechanical and electric devices, three-dimensional silicon structures are being used increasingly. One of the most important problems in VLSI technology (very 
broad integration) is the production of deep trenches. This study demonstrates the spontaneous forming of trenches in n-type silicone immersed in hydrofluoric acid and characterizes the resulting micro structures $[5,6]$.

Black silicon is obtained via two major methods: wet etching of the substrate and the dry etching (plasma etching). The first method involves using chemical solutions (etchants), while the second method deals with laser and plasma etching uses etchant gases to texture the surface [3, 7-10].

The objective of the current study is to manufacture Black silicon structure that is formed by electrochemical etching of p-type Si wafers using electrolytes (hydrofluoric acid and a surfactants). In order to study the behavior of pore formation let's use different current values and different etching times. Adopting chemical etching is advantageous in a fabricating black silicon. Surface morphology indicated that BSI exhibited uniform structure with layered etching phenomena. Etching nature is subject to etching parameters such as the time and current value. Surface morphology is key factor in promoting low reflection values of the etched $\mathrm{Si}$.

\section{Materials and Methods}

In this work, the BSi was obtained by electrochemical etching process. The silicon wafer selected is c-Si of P-type wafer with orientation $<100>$ this wafer cuts to $(1.5 \times 1.5) \mathrm{cm}^{2}$. The thickness of silicon wafer is $(5.50 \mu \mathrm{m})$ with resistivity of $2 \Omega \mathrm{cm}$.

Electrochemical etching is applied for etching process. The chemical solution formed of $\mathrm{HF}$ (Hydrofluoric acid) and $\mathrm{C}_{2} \mathrm{H}_{5} \mathrm{OH}$ (Ethyl Alcohol) at the concentration (1:1). Ethyl Alcohol (surfactant) is often added to facilitate evacuation of $\mathrm{H}_{2}$ bubbles; these bubbles can easily leave the surface because of the decreased in surface tension of the liquid. Additionally, the bubbling enhances the liquid circulation in the electrolization cell, which helps the transport of reactants and side products [3].

The silicon wafer serves as the anode. The cathode is made of platinum (because it is HF-resistant and conducting material). The cell body is made of Teflon (highly acid resistant). The entire silicon wafer serves as the anode, the advantage of this method is its simplicity.

To be able to synthesize layers with high reproducibility, the applied anodic current density and etching time are monitored, controlled, and kept at a particular constant level required during the etching process.

Seven silicon samples where targeted. Each sample was dipped into the electrochemical solution for durations of ( 5 and $25 \mathrm{~min}$ ). The anodic current starts from $0.1,0.2,0.3, \ldots, 0.7 \mathrm{~mA}$ for each sample, as shown as in Fig. 1.

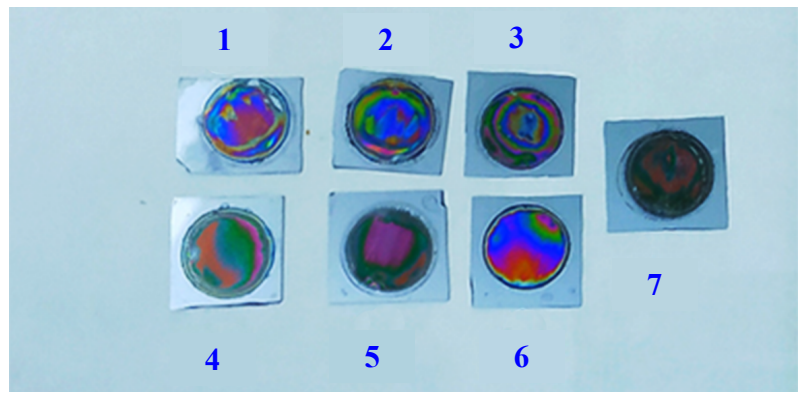

Fig. 1. Many samples of black silicon after etching processing

Seven silicon samples where targeted. Each sample was dipped into the electrochemical solution for durations of (5 and $25 \mathrm{~min}$ ). The anodic current starts from $0.1,0.2,0.3, \ldots, 0.7 \mathrm{~mA}$ for each sample. The devices was used:

1. FESEM(Hitachi. model S-4160 FE-SEM): has been employed to determine the particle size and distribution.

2. UV-win.5.0 spectrophotometer provided by (P G. China): to measure The reflection properties of the black silicon surface were studied by optical reflectance spectroscopy.

3. Edinburgh RF_551 photoluminescence spectrometer: to measure the photoluminescence properties. 


\section{Results and discussion}

\section{1. Surface morphology (FESEM) test}

To study the dependency of the pore dimension and pore geometry, a sample mirror like BSi sample etched for 25 min with different current values $(0.4,0.6$ and $0.7 \mathrm{~mA})$ was examined with the FESEM device. Magnified images are shown in Fig. 2, $\boldsymbol{a}-\boldsymbol{c}$. The pore size is found to range from $20 \mathrm{~nm}$ to $100 \mathrm{~nm}$, these samples showed the least reflection values.

The figures show uniformed etching process resulting in a homogeneous structured sample. A closer look shows that the porous is composed of multilayers and the dense pores are at scattered positions. Fig. 2, $c$ shows some spherical shapes formed during the etching process. It can be seen that the fabricated BSi sample with $0.7 \mathrm{~mA}$ is deeper etched compared to the other two samples.

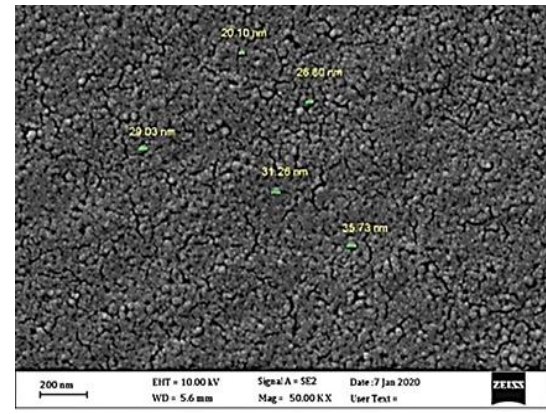

$a$

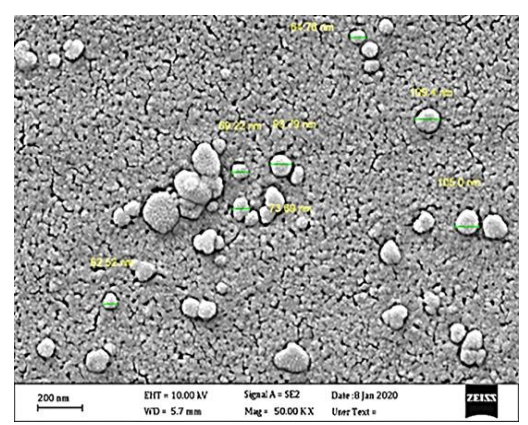

b

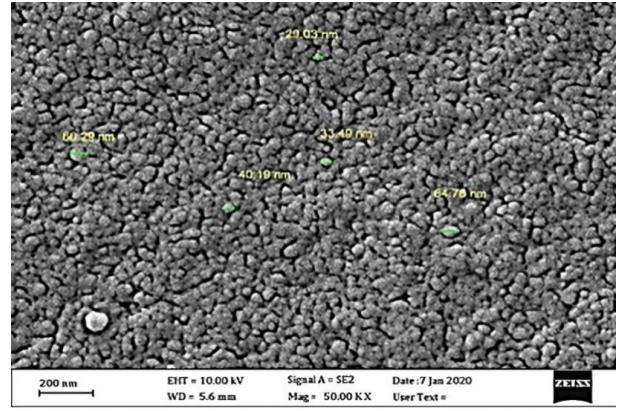

Fig. 2. FESEM images: $a-I=0.4 \mathrm{~mA} ; b-I=0.6 \mathrm{~mA} ; c-I=0.7 \mathrm{~mA}$. Etching time is $25 \mathrm{~min}$

\section{2. Surface reflection of BSi}

Etching time for 5 minutes.

Fig. 3 shows an analysis of reflectance behavior for BSi samples.

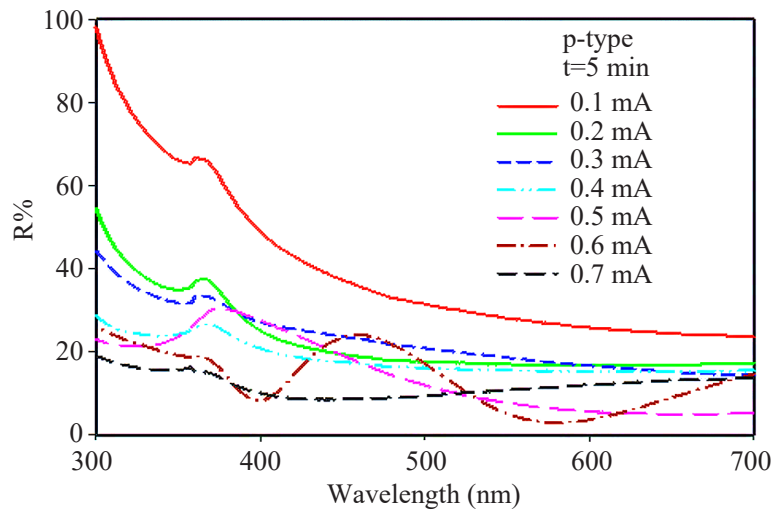

Fig. 3. Normalized reflection spectra for b-S samples electrochemically etched with different current for (5) $\mathrm{min}$

The curves explain the reflectance curves at different etching current values and a constant etching period (5 min). Due to surface morphology of the etched samples, all etched sample showed reduced reflection values. These curves show distinguish response due to the etching current value. Increasing the etching current led to decreasing the reflection value. Results are listed in the Table 1.

Black silicon helps to reduce reflectance for two reasons, depending on the size and shape of its surface texture details: first, there is a reflection reduction because of a multitude of interactions of light with the textured surface; second, when the size of the texture features is large compared to the wavelength of the solar spectrum, surface scattering is responsible for an elongated light path and enhanced absorption [4]. 
Table 1

Reflection analysis for different etching currents and related energy gap values at etching time of (5) minutes

\begin{tabular}{cccc}
\hline Etching current $(\mathbf{m A})$ & Reflection peak $\%$ at $\boldsymbol{\lambda} \mathbf{~ n m}$ & Reflection trough $\%$ vs. $\boldsymbol{\lambda}(\mathbf{n m})$ & Energy gap $(\mathbf{e V})$ \\
\hline 0.1 & 69 at 380 & 38 at 480 & 3.41 \\
0.2 & 38 at 66.5 & 30 at 390 & 3.48 \\
0.3 & 36 at 355.6 & 32 at 420 & 3.38 \\
0.4 & 24 at 377.7 & 19 at 440 & 3.28 \\
0.5 & 22 at 377 & 20 at 439 & 3.29 \\
0.6 & 23 at 459.2 & 11 at 400 , and 5 at 570 & 2.71 \\
0.7 & 19 at 355 & 10 at 410 & 3.49
\end{tabular}

Porous silicon has distinct microstructures with different shapes and heights. The aspect ratio and the effective absorption of the surface increase the number of reflections in the porous silicon layer [5] as explained by Fig. 3.

Fig. 4 is a study of reflection vs. wave length declares the effect of different current values at a constant time duration (25) min. Table 2 depicts BSi reflectance analysis for different etching current values at etching time of (25) $\mathrm{min}$.

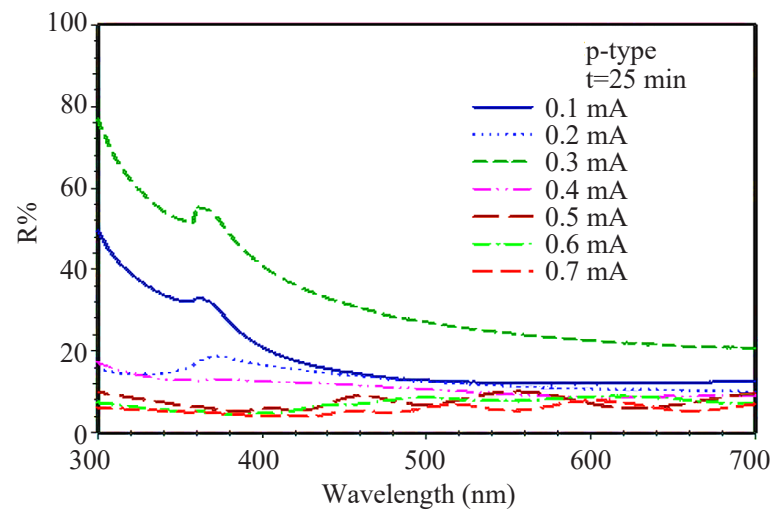

Fig. 4. Reflection spectra for PS samples electrochemically etched with different current at (25) min

Table 2

BSi reflectance analysis for different etching current values at etching time of (25) min

\begin{tabular}{cccc}
\hline Etching current $(\mathbf{m A})$ & Reflection peak $\%$ vs. $\boldsymbol{\lambda} \mathbf{~ n m}$ & Reflection trough $\%$ vs. $\boldsymbol{\lambda}(\mathbf{n m})$ & Energy gap $(\mathbf{e V})$ of BSi \\
\hline 0.1 & 55 at 380.5 & 29.1 at 435 & 3.48 \\
0.2 & 18.2 at 370 & 27.5 at 427 & 3.35 \\
0.3 & 78 at 362.9 & 55.8 at 457 & 3.41 \\
0.4 & 16.6 at 374 & 17.3 at 540 & 3.31 \\
0,5 & 10.5 at 551 & 12.3 at 351 & 3.25 \\
0.6 & 8.5 at 618.5 & 14.1 at 542 & 2 \\
0.7 & 7.3 at 603.7 & 9.6 at 459 & 2.05
\end{tabular}

The maximum reflection value is $78 \%$ at $0.3 \mathrm{~mA}$, and minimum reflection value is equal $7.3 \%$ at $0.7 \mathrm{~mA}$ as shown as in Table 2 declares the reflectivity behavior of samples after etching for 25 minutes at different current values. When comparing the Fig. 3 with the Fig. 4, it was noted that the reflectivity value of the sample in the 0.7 current at time 25 min was $(7.3 \%)$ significantly decreased from the reflectivity value of the same sample and the same current at time $5 \mathrm{~min}$, which was $(19 \%)$. This indicates that the effect of increasing the time in the etching process. It reduces reflectivity. This in turn leads to an increase in the efficiency of the black silicon. 


\section{3. Energy gap calculations}

When the photon energy is at or above the band gap energy, the radiation will be absorbed and a drop in the reflection percentage will be observed. Fig. 5 depicts the relationship between Energy gap and etching current of BSi etched at 5 minutes. On the other hand, Fig. 6 explains the association between etching current and Energy gap of BSi at 25 minutes.

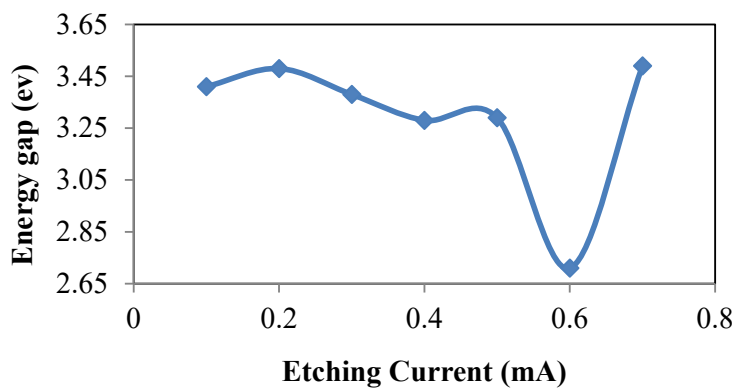

Fig. 5. Relationship between Energy gap and etching current of BSi etched at 5 minutes

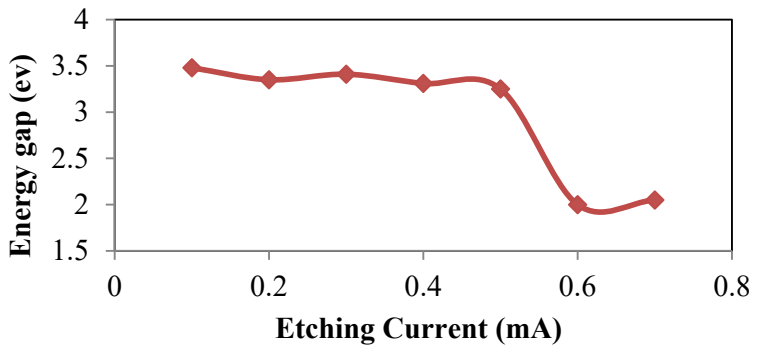

Fig. 6. Relationship between etching current and Energy gap of BSi at 25 minutes

At this wavelength the energy is converted to energy obeying the relation $(E=h c / \lambda)$, so this is the band gap value adopted from Fig. 5, 6 and listed in Tables 1, 2.

\section{4. Photoluminescence Spectroscopy Analysis}

The PL Spectrum, was acquired when the excitation frequency is higher than the emission frequency. This is since higher frequency has higher energy so it causes photoluminescence.

The PS can enlighten light as a result of the surface states and the quantum constringent that created on the BSi after the carving process [7, 8]. From Fig. 7 and Fig. 8, it is declared that the PL power increases with scratching time. This is on the grounds that PL power is influenced by porosity or the all-out volume of crystallites on the outside of PS [9]. The photoluminescence (PL) spectra of the porous silicon for different etching time periods PL signature in a semiconductor consists of near-band-edge emission and defects related luminescence. Near-band-edge emission results from recombination between free electrons (holes) with bound holes (electrons). Fig. 7 describes the relation between wavelength and PL, the figure shows increasing graph vs. wave length until the maximum value (peak) is reached then it decreases after the peak using only 3 points as similar as reported study in [4]. The figure describe PL carves with sharp peak intensity at different wavelength which refereeing to a porous layer as shown as in Table 3. PL band of photoelectrochemical etched Si (110) samples which were prepared at two different current densities. The lower intensity represent etched Si sample which was formed under current density of $26 \mathrm{~mA} / \mathrm{cm}^{2}$ while the higher is for sample under current density of $20 \mathrm{~mA} / \mathrm{cm}^{2}$. These results are consistent with a study of Jayachandran et al. They showed that by increasing the current density up to $20 \mathrm{~mA} / \mathrm{cm}^{2}$, the PL intensity raised and then decreased [11]. More points can be adopted as future prospective work. Black silicon can be as well to be employed as future work with antenna sensors with broadband response as similar to microstrip antenna [12].

The porous samples with different etching current, PL peak value (au) in 25 minutes. The PL peak intensity show maximum valued of about at etching current $0.7 \mathrm{~mA}$. 


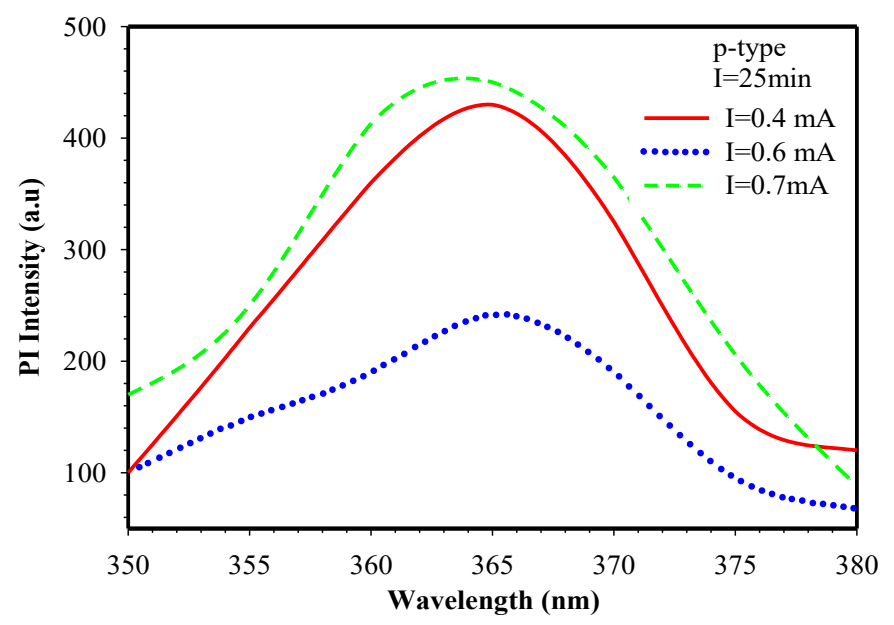

Fig. 7. The relation between wavelength and PL

Table 3

Photoluminescence analysis for BSi at different etching currents, etched for 25 minutes

\begin{tabular}{ccccc}
\hline Etching time (min) & Current $(\mathbf{m A})$ & Wavelength $(\mathbf{n m})$ & PL Peak (au) & Energy gap for $(\mathbf{e V})$ \\
\hline 25 & 0.4 & 355.5 & 450 & 0.03488 \\
25 & 0.6 & 365 & 260 & 0.03398 \\
25 & 0.7 & 364 & 460 & 0.03370
\end{tabular}

This enhancement at PL intensity is generally limited by quantum size effects in nanostructures. The increase in etching time leads to the transformation of some silicon nano-wires to more energized quantum dotes [4].

\section{5. Porosity analysis by Gravimetric Method}

The weight of the sample, before and after etching was measured to determine the weight loss and the porosity $\%$ of the sample. After electrochemical etching, porosity had been studied by using gravimetric measurements and using these equations [10]:

$$
P=(w 1-w 2) /(w 1-w 3)
$$

where $w 1, w 2$ are the weights of sample silicon before and after etching $w 3$ is the weight after removed porous silicon layers.

The obtained porosity \% shows that it was dependent on the etching time as depicted in Fig. 8.

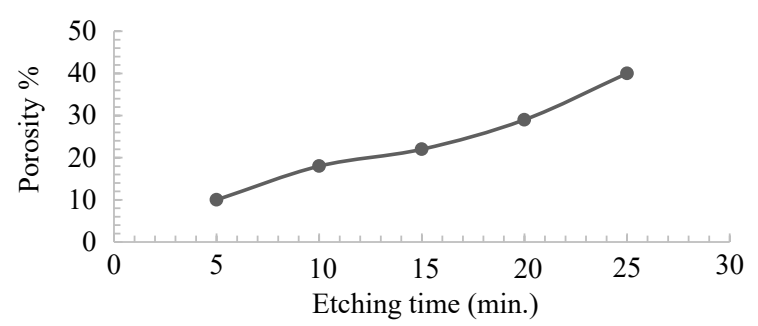

Fig. 8. The Porosity of B Si vs. Etching Time

The porosity of the BSi produced by the chemical etching method is in the range between $(9$ to 40$) \%$. Besides, the etching rate shows a gradual increase with time. This might be due to the hindrance of the bubble produced during the etching process. As bubble formed on the sample surface, it reduces the surface area for etching process for a short moment before the bubble released from the sample surface [6]. 


\section{Conclusions}

The surface morphology indicated that BSI technique exhibited a uniform structure with layered etching phenomena. Etching nature depends on etching parameters such as the time and the current value. Surface morphology is the key factor in promoting low reflection values of the etched $\mathrm{Si}$, increasing the current and or the time led to more etching layers. Thicker and more uniform porous silicon layers with possible higher photoluminescence intensity are obtained via increasing the porosity percentage. The porosity has been within (10-40) \% and it is reliant on etching time and etching current value. Etching density is $0.1073 / \mathrm{mm}^{2}$. Reflection analysis provides the smallest reflectivity spectrum with the intensity of peaks decrease and red shifted with increasing the etching current. A peak of the photoluminescence is about $360 \mathrm{~nm}$, PL peaks have been blue shifted and proportional to etching current. The ranged band gap energy from 2 to $3.4 \mathrm{eV}$, it is affected with porous morphology and directly proportional to etching current magnitudes.

\section{Acknowledgments}

The authors wish to thank the members of thin films applications lab, especially Prof. Dr. Isam M. Ibrahim, in University of Baghdad/College of Science/Department of Physics (Iraq).

\section{References}

[1] Wahab, N. H. A., Rahim, A. F. A., Mahmood, A., Yusof, Y. (2017). Investigation on the structural characterization of pulsed p-type porous silicon. doi: https://doi.org/10.1063/1.4998356

[2] Al-kadumi, A. Kh., Sharif, M. R. (2014). The Fabrication of Porous Silicon by Electrochemical Etching with Photo Assisted. International Journal of Enhanced Research in Science Technology \& Engineering, 3 (11), 167-171.

[3] Ee, D. T. J., Sheng, C. K., Isa, M. I. N. (2011). Photoluminescence of porous silicon prepared by chemical etching method. The Malaysian Journal of Analytical Sciences, 15 (2), 227-231.

[4] Alwan, A. M., Jawad, M. S. M. (2013). The Photoluminescence Characteristics of Partially and Fully (P-N) Porous Silicon. Eng. \&Tech. Journal, 31 (3), 391-399.

[5] Xu, J. (2019). Preparation of Porous Silicon by Electrochemical Etching Methods and its Morphological and Optical Properties. International Journal of Electrochemical Science, 5188-5199. doi: https://doi.org/10.20964/2019.06.10

[6] Lehmann, V., Föll, H. (1990). Formation Mechanism and Properties of Electrochemically Etched Trenches in n-Type Silicon. Journal of The Electrochemical Society, 137 (2), 653-659. doi: https://doi.org/10.1149/1.2086525

[7] Canham, L. T. (1990). Silicon quantum wire array fabrication by electrochemical and chemical dissolution of wafers. Applied Physics Letters, 57 (10), 1046-1048. doi: https://doi.org/10.1063/1.103561

[8] Lehmann, V., Gösele, U. (1991). Porous silicon formation: A quantum wire effect. Applied Physics Letters, 58 (8), $856-858$. doi: https://doi.org/10.1063/1.104512

[9] Kim, D.-A., Shim, J.-H., Cho, N.-H. (2004). PL and EL features of p-type porous silicon prepared by electrochemical anodic etching. Applied Surface Science, 234 (1-4), 256-261. doi: https://doi.org/10.1016/j.apsusc.2004.05.028

[10] Masi, M., Cavallotti, C., Carra, S. (2001). Gas phase and surface kinetics of silicon chemical vapor deposition from silane and chlorosilanes. Silicon-Based Material and Devices, 155-186. doi: https://doi.org/10.1016/b978-012513909-0/50006-4

[11] Amirhoseiny, M., Hassan, Z., Ng, S. S. (2012). Effect of current density on optical properties of anisotropic photoelectrochemical etched silicon (110). Modern Physics Letters B, 26 (20), 1250131. doi: https://doi.org/10.1142/s021798491250131x

[12] Shandal, S., Mezaal, Y. S., Kadim, M., Mosleh, M. (2018). New Compact Wideband Microstrip Antenna for Wireless Applications. Advanced Electromagnetics, 7 (4), 85-92. doi: https://doi.org/10.7716/aem.v7i4.860

How to cite: Hwail, H. M., Abdullah, M. M. (2021). Implementation of P-type black silicon with high aspect ratio for optoelectronics applications. EUREKA: Physics and Engineering, 4, 134-140. doi: https://doi.org/10.21303/2461-4262.2021.001902 